\title{
ARTICLE
}

\section{Evaluation of a novel monoclonal antibody mAb109 by immuno-PET/fluorescent imaging for noninvasive lung adenocarcinoma diagnosis}

Hua Zhu' ${ }^{1,2}$, Te-li Liu' ${ }^{1}$, Chang-hao Liu ${ }^{2}$ Jing Wang ${ }^{2}$, Hong Zhang ${ }^{3}$, Bin Dong ${ }^{3}$, Jing Shen ${ }^{3}$, Chuan-ke Zhao $^{4}$, Zhen-fu Li $^{3}$, Zhen Cheng ${ }^{2}$ and Zhi Yang ${ }^{1}$

Monoclonal antibodies are believed to be magic bullets and hold great potential for lots of biological process. About $100 \mu \mathrm{g}$ of mAb109 was expressed in $5 \times 10^{6}$ cells after 10 days' immunization. ${ }^{64} \mathrm{Cu}-\mathrm{NOTA}$-mAb109 was synthesized with the specific activity of $0.74 \mathrm{MBq} / \mu \mathrm{g}$ and high in vitro stability. The binding affinity of ${ }^{64} \mathrm{Cu}-\mathrm{NOTA}-\mathrm{mAb} 109$ in A549 cells was determined to be $29.64 \mathrm{nM}$. ${ }^{64} \mathrm{Cu}-\mathrm{NOTA}-\mathrm{mAb} 109$ displayed prominent tumor accumulation from $2 \mathrm{~h}$ to $60 \mathrm{~h}$ p.i. $(9.34 \pm 0.67 \%$ ID/g). NIRF imaging of Cy5.5mAb109 showed high accumulation till 9 days p.i., while tumors nearly can not be observed in negative groups, which was confirmed by autoradiography. Immunohistological study confirmed that mAb109 had strong and specific capacity to bind lung adenocarcinoma (concentration to $58 \mathrm{nM}$ ). Our study demonstrated mAb109 was a new platform for the development of novel agent for lung adenocarcinoma noninvasive imaging. The resulted ${ }^{64} \mathrm{Cu}-\mathrm{NOTA}-\mathrm{mAb} 109 / \mathrm{Cy} 5.5-\mathrm{mAb} 109$ show favorable imaging properties/specificity for A549 tumor and high sensitivity to human lung adenocarcinoma tissues.

Keywords: human lung adenocarcinoma; ${ }^{64} \mathrm{Cu}-\mathrm{NOTA}-\mathrm{mAb109}$; antibody production; positron-emission tomography (PET) imaging; NIRF imaging

Acta Pharmacologica Sinica (2020) 41:101-109; https://doi.org/10.1038/s41401-019-0294-9

\section{INTRODUCTION}

Lung cancer is the most common cancer and the leading cause of cancer death in China over the past 20 years [1]. According to data from the National Central Cancer Registry (NCCR), adenocarcinoma has replaced squamous cell carcinoma as the most predominant histological subtype of lung cancer in China, with an incidence of nearly $43.4 \%$ among male lung cancer patients in 2012 , consistent with the incidence of $40 \%$ in the United States [2-4]. Advanced imaging techniques play essential roles in lung cancer diagnosis, staging, and follow-up.

${ }^{18} \mathrm{~F}$-fluorodeoxyglucose ( $\left({ }^{18} \mathrm{~F}-\mathrm{FDG}\right)$ PET imaging is conventionally used to perform real-time functional imaging of lung carcinoma. However, the ${ }^{18} \mathrm{~F}$-FDG tracer also has inherent deficiencies due to its limited specificity [5]. For example, ${ }^{18}$ F-FDG has relatively low uptake in pulmonary adenocarcinoma, which may lead to a falsenegative diagnosis. Moreover, ${ }^{18} \mathrm{~F}$-FDG PET has low sensitivity and often misses small malignant lesions between 2 and $5 \mathrm{~mm}$ in diameter [6]. To improve the detection sensitivity and specificity of ${ }^{18}$ F-FDG PET for lung tumors, a large number of tracers have been developed. Due to the benefits of their excellent antigen-specific and binding affinity, monoclonal antibodies are believed to be magic bullets and hold great potential for both tumor imaging and therapy [7].

Peroxiredoxin-I (Prdx I) is a member of the redox-regulating protein family peroxiredoxins, which play essential roles in oxidative stress and cell signaling [8]. The Prdx-1 protein is located in the cell membrane and encoded by the Prdx I gene in humans, which is highly expressed in various solid tumors, especially lung tumors [9-11]. Chang et al. [12] confirmed the increased expression of Prdx I by comparing cancer tissues with normal tissues of lung cancer patients and suggested that Prdx I is a useful biomarker for lung cancer. Kim et al. [13] evaluated the expression of Prdx1 and Nrf2 in 90 patients who underwent curative surgical resection and indicated that $\operatorname{Prdx} I$ is an independent prognostic factor for lung cancer. Recently, Jiang et al. found that Prdx1 and Prdx4 were preferentially expressed in human lung cancer, including squamous cell carcinoma and adenocarcinoma [14]. These results revealed a statistically significant correlation among the expression of Prdx 1 and lung tumor cell proliferation, recurrence and progression in patients. Therefore, we hypothesize that Prdx 1 holds great potential as an ideal target for lung tumor diagnosis and therapy.

\footnotetext{
${ }^{1}$ Key Laboratory of Carcinogenesis and Translational Research (Ministry of Education/Beijing), Department of Nuclear Medicine, Peking University Cancer Hospital \& Institute, Beijing 100142, China; ${ }^{2}$ Molecular Imaging Program at Stanford (MIPS), Bio-X Program, Department of Radiology, Stanford University, Stanford, CA CA94305, USA; ${ }^{3}$ Key Laboratory of Carcinogenesis and Translational Research (Ministry of Education/Beijing), Central Laboratory, Peking University Cancer Hospital \& Institute, Beijing 100142, China and ${ }^{4}$ Key Laboratory of Carcinogenesis and Translational Research (Ministry of Education/Beijing), Department of Biochemistry and Molecular Biology, Peking University Cancer Hospital \& Institute, Beijing 100142, China
}

Correspondence: Zhen Cheng (zcheng@stanford.edu) or Zhi Yang (pekyz@163.com)

These authors contributed equally: Hua Zhu, Te-li Liu

Received: 17 March 2019 Accepted: 17 July 2019

Published online: 18 September 2019 
However, the significance of Prdx I in lung cancer progression and recurrence has not been fully investigated. We previously reported the preliminary results of ${ }^{111}$ In-labeled mAb109 antibody for the imaging of Prdx I overexpression tumor [15]. PET nuclides are another good choice for acquiring high-quality images. ${ }^{64} \mathrm{Cu}$ is a well-established radionuclide that can be used for PET imaging and targeted radiotherapy of tumors [16]. ${ }^{64} \mathrm{Cu}$-labeled monoclonal antibodies have a strong binding affinity and specificity for their cognate antigen $[17,18]$, which can be effectively detected by immuno-PET equipment.

In this study, we describe the production and characterization of a novel antibody, namely $\mathrm{mAb109}$, for use in noninvasive lung tumor diagnosis. A modified method of mAb109 production was developed, and characterization of this antibody was conducted. Then, the mAb109 antibody was used as the primary antibody to confirm its sensitivity to lung tumor. Moreover, a near infrared fluorescence (NIRF) chromophore group, Cy5.5, was conjugated to mAb109 for long-period imaging. In addition, the mAb109 antibody was modified by NOTA and radiolabeled with ${ }^{64} \mathrm{Cu}$ for noninvasive positron-emission tomography (PET) imaging of lung tumors. Finally, immunohistological analysis of 12 lung adenocarcinomic carcinoma tissue samples was performed using mAb109 to evaluate its potential for detecting human lung adenocarcinoma.

\section{MATERIALS AND METHODS}

Reagents and cell culture

The details of the reagents and cell culture are provided in the Supplemental Materials. S-2-(4-isothiocyanatobenzyl)-1,4,7triazacyclononane-1,4,7-triacetic acid (p-SCN-Bn-NOTA) was purchased from Macrocyclics, Inc. (Plano, TX, USA). Chelex 100 resin (50-100 mesh) was purchased from Sigma-Aldrich (St. Louis, MI, USA). PD-10 desalting columns were purchased from GE Healthcare (Chicago, IL, USA). Prdx1 was acquired from Sinobiological (Beijing, China). All other reaction buffers and chemicals were purchased from Thermo Fisher Scientific (Fisher store in Stanford, USA).

Generation, characterization, NOTA conjugation, and ${ }^{64} \mathrm{Cu}$ radiolabeling of the monoclonal antibody

The human breast carcinoma ZR 75-1 cell line was purchased from ATCC. Female BALB/C mice (6-8 weeks) were intraperitoneally (i.p.) immunized twice with $5 \times 10^{6}$ ZR75-1 cells (every 3 weeks). Four days later, splenocytes were harvested. B cells were fused with SP2/0 mouse myeloma cells and cultured in hypoxanthine, aminopterin, and thymidine (HAT medium). The conditioned medium for each clone was screened for anti ZR75-1 $\mathrm{mAb}$ presentation using ELISA. For rounds of subcloning/screening, which included lymph cells, ABO red cells, marrow cells, and fibroblast cell negative reactions, were performed. The selected clones, namely, mAb109, were cultured in PRMI-1640 medium under normal cell culture conditions. A total of $5 \times 10^{6} \mathrm{mAb109}$ cells were intraperitoneally inoculated into BALB/C mice, and ascites fluid was collected after 10 days. Monoclonal antibodies were purified with $\mathrm{CNBr}$-activated Sephrose4B-protein A chromatography.

The purity of mAb109 was higher than $95 \%$ as analyzed by HPLC and sodium dodecyl sulfate polyacrylamide gel electrophoresis (SDS-PAGE; $5 \%$ stacking gel, and $8 \%$ resolving gel). The molecular weight of mAb109 was evaluated by matrix-assisted laser desorption/ionization (MALDI) analysis. The spectrum was acquired using a positive linear model and analyzed using FlexAnalysis v3.0 software.

mAb109 or a nonspecific IgG antibody was conjugated with NCS-Bz-NOTA at a molar ratio of $1: 10$ and $\mathrm{pH}$ of 8.3 for 4 $\mathrm{h}$ as previously reported [15]. The final products (NOTA-mAb109) were purified by PD-10 size-exclusion columns using phosphate- buffered saline (PBS) as the mobile phase to remove unbound NCS-Bz-NOTA. The conjugation of Cy5.5 to mAb109 or a nonspecific IgG antibody was similar (Fig. S3). ${ }^{64} \mathrm{Cu}$-NOTAmAb109 and ${ }^{64} \mathrm{Cu}-$ NOTA-lgG were radiosynthesized and purified using reported methods [18].

Cell uptake and binding affinity studies

For the fluorescence microscopy study, A549 cells $\left(1.0 \times 10^{5}\right)$ were plated on $35 \mathrm{~mm}$ MatTek glass bottom culture dishes. After $24 \mathrm{~h}$, the cells were washed with PBS and then incubated with Cy5.5mAb109 ( $55 \mathrm{nM})$ at $37^{\circ} \mathrm{C}$ for $3 \mathrm{~h}$. After incubation, the cells were washed with cold PBS $(2 \mathrm{~mL} \times 3)$. The fluorescence signal of the cells was recorded using an Axiovert $200 \mathrm{M}$ fluorescence microscope (Carl Zeiss Microlmaging, Inc.) equipped with the Cy5.5 filter set (exciter, HQ 650/20 nm; emitter, HQ 695/35 nm).

For the cell uptake study of ${ }^{64} \mathrm{Cu}$-NOTA-mAb109, A549 cells were plated on 24 -well plates $\left(1.0 \times 10^{5}\right.$ cells/well) and incubated overnight. Cells were washed twice with serum-free medium and incubated with a radiotracer $(2 \mu \mathrm{Ci}$ per well, $74 \mathrm{kBq})$ in $1 \mathrm{~mL}$ of serum-free medium at $37^{\circ} \mathrm{C}$. At $15,30,60$, and $120 \mathrm{~min}$, cells were washed with cold PBS $(0.5 \mathrm{~mL} \times 2)$ and lysed with $0.5 \mathrm{~mL}$ of $0.2 \mathrm{M} \mathrm{NaOH}$.

The cell binding affinity assay for ${ }^{64} \mathrm{Cu}-\mathrm{NOTA}-\mathrm{mAb} 109$ was conducted with human adenocarcinoma A549 cells. Cells $\left(1 \times 10^{4}\right.$ cells/well) were plated on 96 -well plates $24 \mathrm{~h}$ before the test. Cells were washed with PBS $(0.1 \mathrm{~mL} \times 2)$. Serum-free Dulbecco's modified Eagle's medium $(0.1 \mathrm{~mL})$ was added to each well. A probe $(0.333-66.7 \mathrm{nM}$ final concentration) was added to the wells. To study the binding specificity, increasing amounts of cold, unlabeled mAb109 were added, and the cells were incubated at $37{ }^{\circ} \mathrm{C}$ for $4 \mathrm{~h}$. Then, the cells were washed with cold PBS $(0.1 \mathrm{~mL} \times$ 3) and detached with TrypLE-Express (Invitrogen).

The radioactivity of the cells was measured by a $\gamma$-counter. The binding affinity and the number of accessible binding sites were estimated by nonlinear regression analysis with a 1-site total binding model using the software package GraphPad Prism (GraphPad Software, Inc.). The concentration at which half of binding was prevented by the blocking agent $\left(\mathrm{IC}_{50}\right)$ was calculated by nonlinear regression with a 1-site competition model with a variable Hill slope using GraphPad Prism.

Small-animal PET, biodistribution studies, and autoradiography of ${ }^{64}$ Cu-NOTA-mAb109

All animal studies were conducted under a protocol approved by the Stanford University Institutional Animal Care and Use Committee. A549 tumor models were prepared with a previously reported method. To generate subcutaneous xenografts, $1 \times 10^{7}$ A549 cells were suspended in $100 \mu \mathrm{L}$ of Dulbecco's phosphatebuffered saline (Invitrogen Life Technologies, Carlsbad, CA) and subcutaneously injected near the left or right forelimb of adult female athymic nude mice (4 week old, Charles River Laboratories, Inc., Cambridge, MA). Imaging was performed when tumors grew to $0.5-1.0 \mathrm{~cm}$ in diameter.

PET imaging and image analysis were performed using an R4 PET rodent scanner (Siemens Medical Solutions) as previously reported [17]. After all of the PET scans had been performed, animals were sacrificed and the blood, heart, and other major organs were collected, weighed and measured for radioactivity using a $\gamma$-counter (Packard Instruments). The results are presented as \%ID/g. Values are expressed as the mean \pm SD for four animals. After imaging, sections $(10 \mathrm{~mm})$ were generated from snap-frozen tumor tissue harvested from A549 xenografts mice. The sections were stained with hematoxylin and eosin to confirm the histologic status or placed on storage phosphor screens to obtain autoradiography images of the tumors.

Autoradiography analysis was performed as previously reported [18]. Mice were sacrificed at $60 \mathrm{~h}$ after micro-PET imaging of ${ }^{64} \mathrm{Cu}-$ NOTA-mAb109, and then, tumor tissues were removed and stored 
at $-20^{\circ} \mathrm{C}$ for $10 \mathrm{~min}$. The frozen organ was then cut into approximately $1 \mathrm{~mm}$ slices and placed onto the surface of a phosphor screen (Amersham Biosciences). The screens were kept in dark for 5 days and were then screened using Tyhoon TRIO equipment.

\section{Fluorescent imaging of Cy5.5 conjugates}

The synthesis and characterization of the Cy5.5-antibody conjugates are shown in Fig. S3. In vivo fluorescence imaging was performed with an IVIS 200 small-animal imaging system (Xenogen, Alameda, CA). For the positive control experiment, mice $(n=4)$ were injected with $0.1 \mathrm{nmol}$ of Cy5.5-mAb109 via the tail vein and subjected to optical imaging at various timepoints (from $2 \mathrm{~h}$ to 2 weeks). For the blocking experiment, mice $(n=3)$ were injected with a mixture of $1.5 \mathrm{mg}$ (100 eq excess) of mAb109 and $0.1 \mathrm{nmol}$ of Cy5.5-mAb109. For the negative control experiments, mice $(n=4)$ were injected with $0.1 \mathrm{nmol}$ of Cy5.5-lgG. IVIS$200 \mathrm{NIR}$ fluorescent images were acquired using a 3-s exposure time, $f /$ stop $=4$, and binning $=4$. The tumor-targeting efficacy of modified Cy5.5-mAb109 was also evaluated by static small-animal NIRF scans of mice bearing human lung cancer A549.

Immunohistological study of human lung tumor tissues All specimens were collected during surgery after obtaining written informed consent from patients, and their use for immunohistochemical staining was approved by the Ethics Committee of Peking University Cancer Hospital, China. Lung tumor specimens were surgically removed from patients, and their classification was determined by two or more pathologists. Immunohistochemistry analysis of each sample was performed as previously described using mAb109 as the primary antibody. Immunostaining was performed using the PV-9000 (2-step plus ${ }^{\circledR}$ Poly-HRP AntiMouse/Rabbit lgG detection system) protocol. The intensity of staining was graded as follows: 0 , no signal; 1 , weak staining; 2 , moderate staining; and 3 , strong staining.

\section{Statistical analysis}

Statistical analysis was performed using Student's $t$ test for unpaired data. The $95 \%$ confidence level was chosen to determine significance between groups, with a $P$ value less than 0.05 being significantly different.

\section{RESULTS}

Chemistry and radiochemistry

Antibody production: To generate a high level of the anti-human mAb109 monoclonal antibody, as shown in Fig. 1a, we adopted a fast immunization approach that consisted of preparing cell hybridomas from B cells harvested from the popliteal lymph nodes of mice immunized with the human breast cancer ZR75-1 cell line. The mAb109 antibody was collected and purified through a PD-10 desalting size-exclusion column (GE Healthcare Life Sciences) (Exclusion limit, $5000 \mathrm{Da}$ ) using phosphate-buffered saline (PBS) as the mobile phase before modification.

There was one clear band at $150 \mathrm{kDa}$ on SDS-PAGE under nonreducing conditions, which represented the bioactive mAb109, and its purity was over $80 \%$. There were two bands at 50 and $25 \mathrm{kDa}$, corresponding to the heavy chain and light chain, respectively, on the same SDS-PAGE slice under reducing conditions. MALDI-TOF mass spectrometry confirmed that the molecular weight of mAb109 was 147128.4 Da (Fig. 1b). The expression of Prdx1 in A549 cancer cells was measured by Western blot analysis, and the results are shown in Fig. 1c, d.

Modification and in vitro evaluation of the mAb109 antibody Cy5.5-mAb109 was synthesized by conjugating the mAb109 antibody with Cy5.5-NHS using standard coupling chemistry, followed by purification through a PD-10 column. To radiolabel
mAb109 for PET imaging using ${ }^{64} \mathrm{Cu}\left(T_{1 / 2}=12.7 \mathrm{~h}\right)$ as the radioisotope, $\mathrm{mAb} 109$ was conjugated to the bifunctional chelator NCS-Bz-NOTA using standard coupling chemistry at a high yield $(>60 \%)$, radiochemical purity (>95\%) (Fig. S1), and specific activity $(\sim 0.74-1.48 \mathrm{MBq} / \mathrm{\mu g})$. The contrast tracer ${ }^{64} \mathrm{Cu}-\mathrm{NOTA}-\mathrm{lgG}$ was prepared using the same process.

Cell uptake of ${ }^{64} \mathrm{Cu}-\mathrm{NOTA}-\mathrm{mAb}$ in $\mathrm{A} 549$ cells increased over time. ${ }^{64} \mathrm{Cu}$-NOTA-mAb109 with excess mAb109 and ${ }^{64} \mathrm{Cu}$-NOTAIgG had a similar uptake in A549 cells (Fig. 2b). The cell uptake values of ${ }^{64} \mathrm{Cu}$-NOTA-mAb109 at $15,30,60$, and $120 \mathrm{~min}$ and $37^{\circ} \mathrm{C}$ were $3.04 \% \pm 0.34 \%, 3.78 \% \pm 0.27 \%, 4.65 \% \pm 0.28 \%$, and $6.77 \% \pm$ $0.09 \%$, respectively. In the block groups, the cell uptake values were $1.95 \% \pm 0.33 \%$ and $2.33 \% \pm 0.30 \%$ at 60 and $120 \mathrm{~min}$, respectively. A threefold greater accumulation of the probe occurred in cells incubated at $37^{\circ} \mathrm{C}$ compared with the block group, indicating that cell surface binding could be significantly inhibited by the presence of excess mAb109 $(P<0.001)$.

The affinity of ${ }^{64} \mathrm{Cu}$-NOTA-mAb109 was tested by binding to A549 cells. Competition binding and saturation studies demonstrated that ${ }^{64} \mathrm{Cu}$-NOTA-mAb109 specifically bound to A549 cells with nanomolar affinity. The $B_{\max }$ value was $354 \mathrm{fmol} / 6000$ A549 cells. The affinity of ${ }^{64} \mathrm{Cu}$-NOTA-mAb109 was $29.6 \mathrm{nM}$, which was tested by a one site saturation binding assay (Fig. 2c). As shown in Fig. 2d, blocking the specific binding of ${ }^{64} \mathrm{Cu}-\mathrm{NOTA}-\mathrm{mAb} 109$ to its receptor by adding serial increasing amounts of cold, unlabeled mAb109 indicated that it had a good specificity of $10.6 \mathrm{nmol}$ $\left(\log \left[I C_{50}\right]\right)$.

\section{Micro-PET/CT imaging and biodistribution study}

The tumor-targeting efficacy of ${ }^{64} \mathrm{Cu}$-NOTA-mAb109 was evaluated by static micro-PET/CT scanning in human adenocarcinoma A549 tumor-bearing mice. Representative decay-corrected coronal PET/CT images obtained at different time points are shown in Fig. 3. Blocking experiments were also conducted using $100 \mu \mathrm{g}$ of cold mAb109 coinjected with ${ }^{64} \mathrm{Cu}$-NOTA-mAb109. ${ }^{64} \mathrm{Cu}$-NOTA-IgG was selected as the negative control for a sideby-side comparison, and these results are shown in Fig. S2. As early as $4 \mathrm{~h}$ after administration, tracer uptake in the A549 tumor xenografts was discernible. A549 tumors were clearly visualized in the ${ }^{64} \mathrm{Cu}$-NOTA-mAb109 group $24 \mathrm{~h}$ after injection, with a good tumor-to-background contrast at late time points. Coronal PET images intersecting the tumor showed excellent delineation of the tumor mass with a high tumor/background contrast. In vivo micro-PET images of ${ }^{64} \mathrm{Cu}$-NOTA-mAb109 also displayed significantly higher $(P<0.05)$ tumor accumulation (ROI analysis showed it up to $9.5 \% \mathrm{ID} / \mathrm{g}$ ) than that in the blocked and ${ }^{64} \mathrm{Cu}$ NOTA-lgG groups.

To further validate the PET quantification, the biodistribution by direct tissue sampling was determined after micro-PET/CT imaging at $60 \mathrm{~h}$ after injection (Fig. 3a). The biodistribution study revealed that ${ }^{64} \mathrm{Cu}$-NOTA-mAb109 displayed prominent tumor accumulation from 4 to $60 \mathrm{~h}$ after injection $(9.34 \pm 0.67 \% \mathrm{ID} / \mathrm{g})$. At the same time, tumor uptake in the blocking and ${ }^{64} \mathrm{Cu}-\mathrm{NOTA}$-lgG groups was $2.29 \pm 0.47$ and $2.68 \pm 0.69 \% \mathrm{ID} / \mathrm{g}$, respectively. By contrast, uptake in other normal organs did not show a significant difference between the ${ }^{64} \mathrm{Cu}$-DOTA-mAb109 and blocking groups (Fig. 3b1). Moreover, ${ }^{64}$ Cu-NOTA-mAb109 exhibited the highest tumor-to-lung ratio (3.7) compared with those of the blocking and ${ }^{64} \mathrm{Cu}-\mathrm{NOTA}-\mathrm{IgG}$ groups (1.0) at $60 \mathrm{~h}$ after injection (Fig. 3b2) $(P<0.05)$. The biodistribution results were consistent with the micro-PET/CT quantification.

Micro-PET/CT imaging demonstrated the effectiveness of ${ }^{64}$ Cu-NOTA-mAb109 as a noninvasive imaging agent for the detection of human lung adenocarcinoma A549 tumors in vivo. As shown in Fig. $3 c$, autoradiography of the ${ }^{64} \mathrm{Cu}$ antibody also confirmed this finding. ${ }^{64} \mathrm{Cu}$-NOTA-mAb109 had an obliviously higher accumulation in A549 tumors than the blocked and ${ }^{64} \mathrm{Cu}$ NOTA-IgG groups. 
a

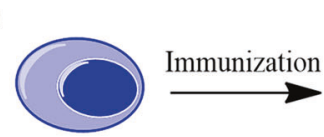

ZR 75 Cells

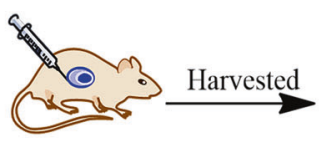

In-vivo ZR75

immunization 2 times

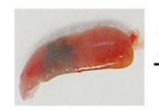

Spleen cell collection

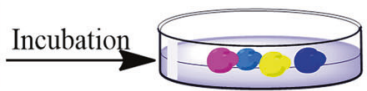

Fusion with mice myeloma SP 2/0
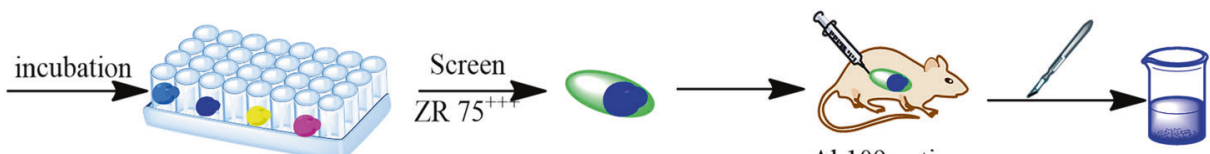

mAb109 antigen immunization

Ascites

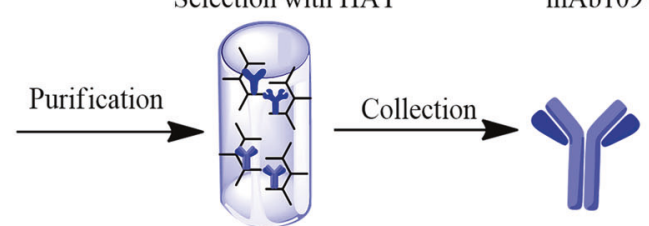

Column Purification mAbl09 antibody

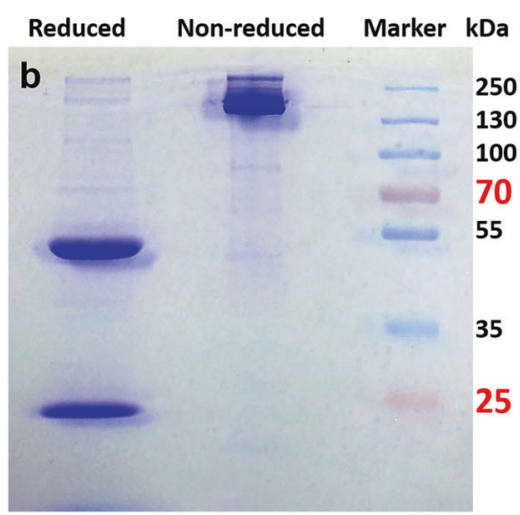

PAGE gel of recombinant mAb109

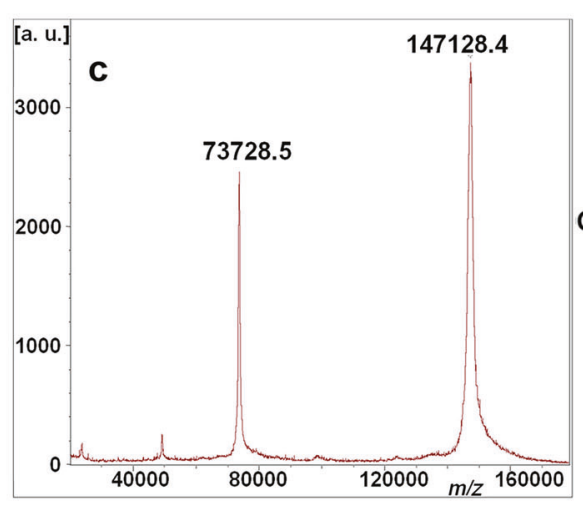

MALDI-TOF analysis of recombinant mAb109 d

A549

GAPDH

Prdx1

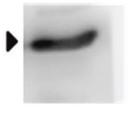

Fig. 1 Expression, purification of and characterization of mAb109. a Schematic representation of mAb109 production process. $\mathbf{b}$ Reduced and nonreduced SDS-PAGE (8\%) analysis of purified mAb109. Molecular weight markers are shown on right. c MALDI-TOF analysis of purified mAb109 antibody. d Western blots of Prdx1 in A549 tumor cells
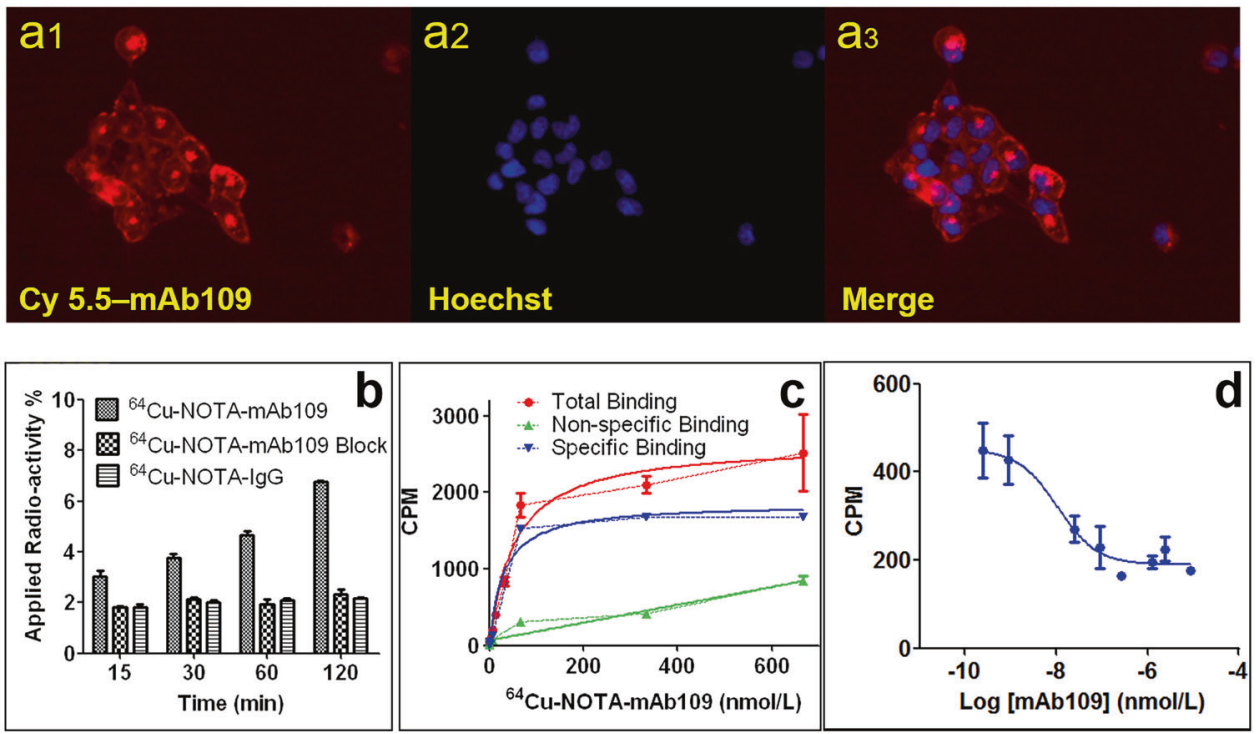

Fig. 2 Evaluation of mAb109 antibody. a Cell uptake of Cy5.5-mAb109 $(55 \mathrm{nM})$ in A549 cells at $37^{\circ} \mathrm{C}$. a1 indicates the NIR fluorescent; a2 indicates the hoechst stain of the live cells, and $\mathbf{a} 3$ indicates the merge of the fluorescent imaging and cell nuclide. Original magnification $\times 20$ object. $\mathbf{b}$ In vitro A549 cell uptake assay. Cell uptake data were expressed as the percentage of the applied radioactivity per total radioactivity. c One site saturation binding study, A549 cells were exposed for $4 \mathrm{~h}$ at $37^{\circ} \mathrm{C}$ to increasing concentrations of ${ }^{64} \mathrm{Cu}-\mathrm{NOTA}-\mathrm{mAb} 109$. d One site competitive binding study, with the increasing amounts of unlabeled mAb109 were used to block binding of ${ }^{64}$ Cu-NOTA-mAb109 to A549 cells 

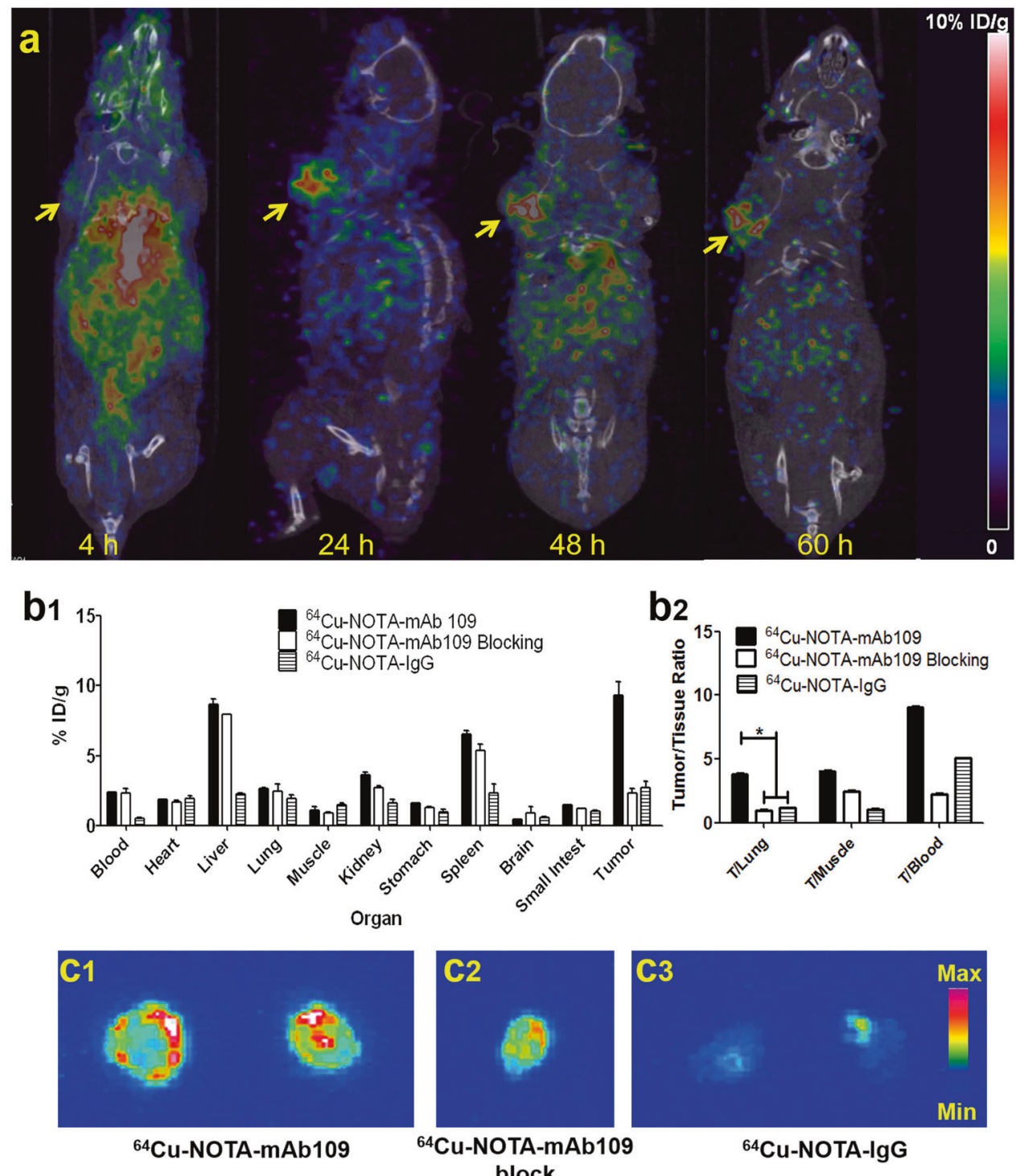

Fig. 3 Micro-PET/CT imaging and biodistribution evaluation of ${ }^{64} \mathrm{Cu}$-NOTA-mAb109. a Decay-corrected whole-body coronal micro-PET/CT static scans in mice bearing A549 tumors at 4, 24, 48, and $60 \mathrm{~h}$ after injection. b1 Biodistribution of ${ }^{64} \mathrm{Cu}-\mathrm{NOTA}-\mathrm{mAb} 109$ without and with $100 \mu \mathrm{g}$ of cold mAb109 or ${ }^{64} \mathrm{Cu}-\mathrm{NOTA}-\mathrm{hlgG}$ in mice bearing A549 tumors at $60 \mathrm{~h}$ after injection, b2 tumor to main organ (selected) ratios. The autoradiography of ${ }^{64} \mathrm{Cu}-\mathrm{NOTA}-\mathrm{mAb} 109$ (c1), ${ }^{64} \mathrm{Cu}-\mathrm{NOTA}-\mathrm{mAb} 109$ coinjection with $100 \mu \mathrm{g}$ cold mAb109 (c2), and ${ }^{64} \mathrm{Cu}-\mathrm{NOTA}-\mathrm{lgG}$ (c3). ${ }^{*} P<0.05$; arrows indicate tumors

NIRF imaging and ROI-based analysis

Figure 4 shows typical NIR fluorescent images in nude mice bearing A549 tumors after an intravenous injection of $0.2 \mathrm{nmol}$ of Cy5.5-mAb109 without (Fig. 4a) or with (Fig. 4b) excess mAb109 and Cy5.5-lgG (Fig. 4c). The A549 tumors could be clearly visualized by the Cy5.5-mAb109 tracer from $24 \mathrm{~h}$ to 16 days. Both the blocked group and the Cy5.5-lgG tracer injected group did not show such persistent and clear NIRF imaging. Moreover, the location of the A549 tumors was evaluated by bioluminescence imaging, as shown in Fig. 4 (right column), 9 days after injection.

Quantification analysis of the ROls was performed, and the tumor-to-normal tissue ratio $T / N$ as a function of time is shown in Fig. 4d. Cy5.5-mAb109 exhibited fast and persistent tumor-targeting properties in vivo. The tumor-to-normal tissue ratio reached 3.2 at $24 \mathrm{~h}$ after injection. The T/N ratios remained over 3.0 for the following seven days. The receptor specificity of Cy5.5-mAb109 was verified by mAb109 blocking and Cy5.5-lgG (negative control) fluorescence experiments. Unlabeled mAb109 significantly reduced tumor uptake and tumor contrast after $24 \mathrm{~h}$. Tumor contrast quantified by ROI analysis of images indicated that the $T / N$ value at $24 \mathrm{~h}$ p.i. was reduced from $3.2 \pm 0.5$ to $2.1 \pm 0.5 \quad(P<0.05)$. This phenomenon was also confirmed by the injection of Cy5.5-lgG as a control. Fluorescent imaging of A549 tumors showed a much lower $T / N$ value $(1.8 \pm 0.3)$. Liver-to-normal tissue $(\mathrm{L} / \mathrm{N})$ quantification analysis of the ROls was also conducted (Fig. 4e). Both Cy5.5-mAb109 (with or without blocker) and IgG had almost the same $\mathrm{L} / \mathrm{N}$ ratios at each time point. For example, the $\mathrm{L} / \mathrm{N}$ ratios of Cy5.5-mAb109 were $2.5 \pm 0.3,1.6 \pm 0.3,1.5 \pm 0.3,1.7 \pm 0.2$, and $1.5 \pm 0.1$ compared with $2.3 \pm 0.4,1.8 \pm 0.4,1.7 \pm 0.4,1.6 \pm 0.3,1.4 \pm$ 0.2 for Cy5.5-lgG at $2 \mathrm{~h}, 1 \mathrm{~d}, 2 \mathrm{~d}, 5 \mathrm{~d}$, and $7 \mathrm{~d}$ after injection, respectively $(P>0.5)$.

The ex vivo imaging results of Cy5.5-mAb109 in A549 tumor mice at $48 \mathrm{~h}$ p.i. are shown in Fig. 5a. Quantitative analysis showed that a large amount of probe was trapped inside tumors and the liver without clearance (Fig. 5c). The tumor-to-normal organ ratios, except the liver ratio, were also high (Fig. 5b). For example, the ratios for tumor-to-blood and tumor-to-kidney were $7.7 \pm 0.7$ and $4.5 \pm 0.4$, respectively. Ex vivo NIRF imaging of tumor-bearing mice 

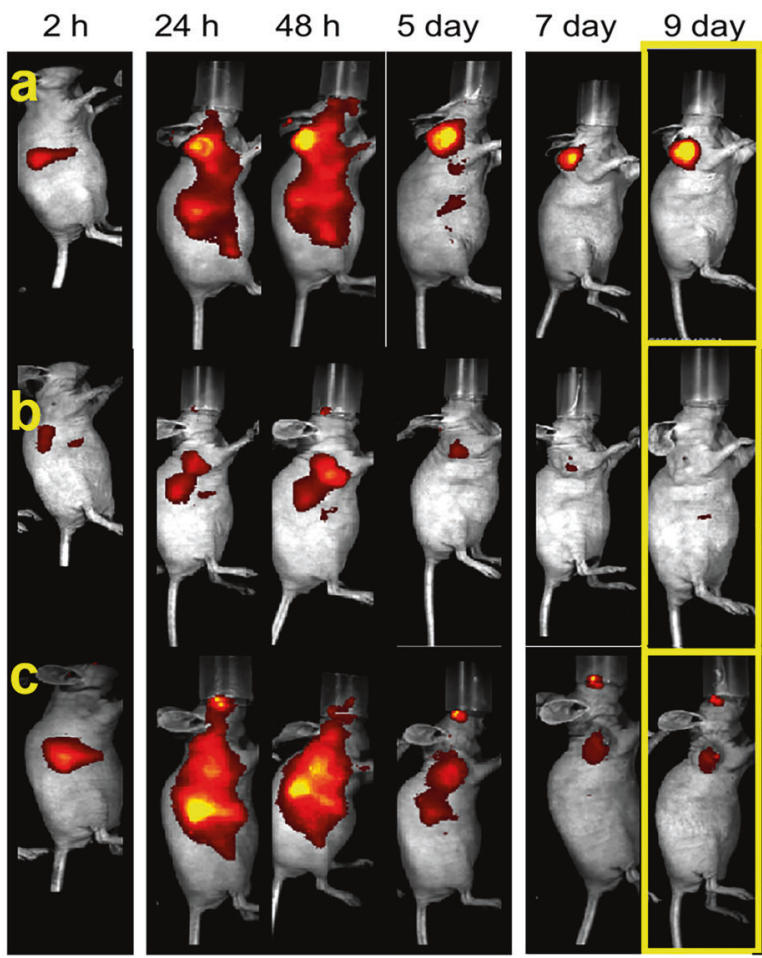

16 day
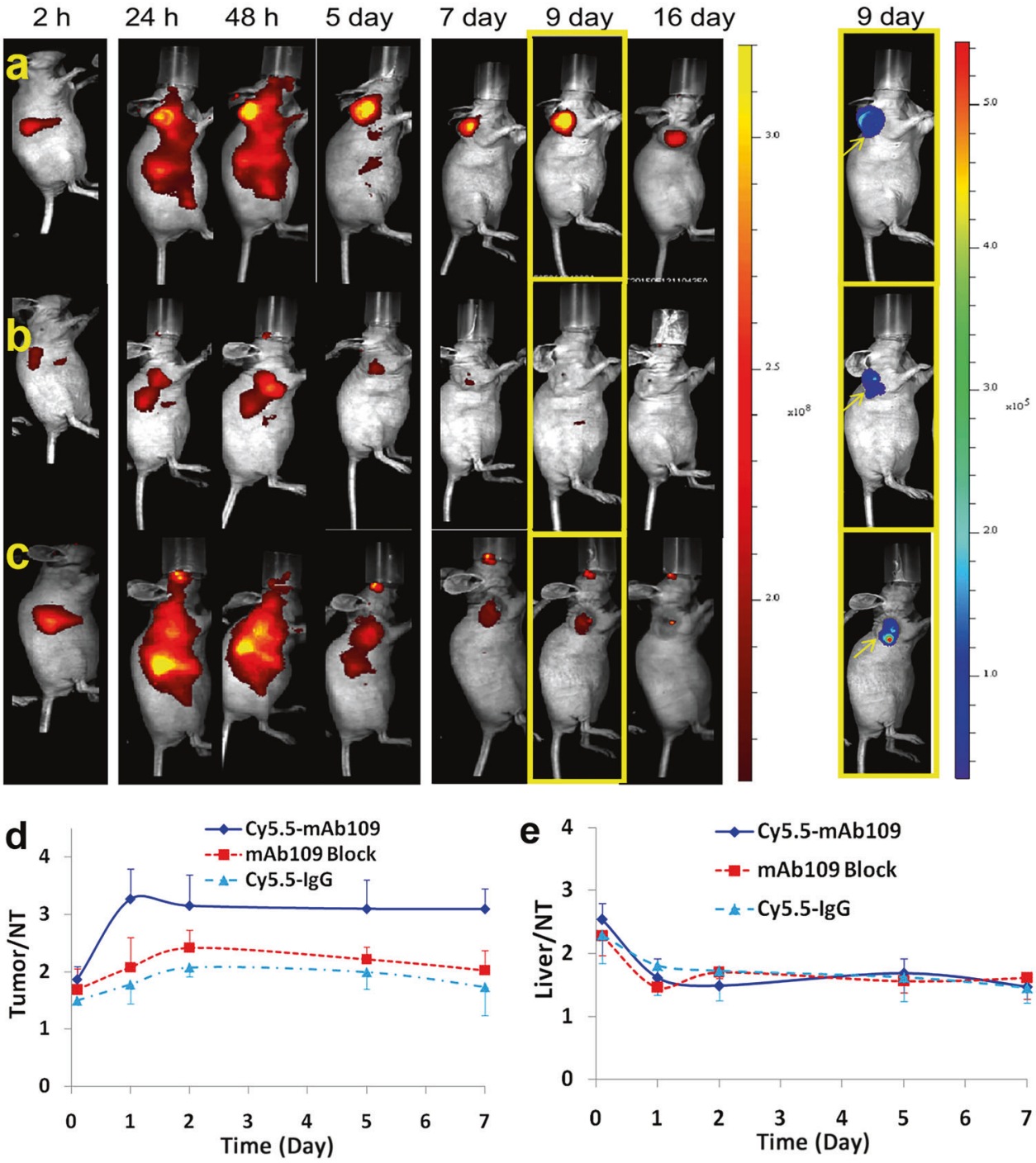

Fig. 4 In vivo fluorescence IVIS-200 imaging of subcutaneous Luci-A549 tumor-bearing nude mice at $2 \mathrm{~h}, 24 \mathrm{~h}, 48 \mathrm{~h}, 5 \mathrm{~d}, 7 \mathrm{~d}, 9 \mathrm{~d}$, and $16 \mathrm{~d}$ of Cy5.5-mAb109 (0.2 nmol) (a), with (middle) coinjection of unlabeled mAb109 (500 $\mu \mathrm{g})(\mathbf{b})$, and Cy5.5-lgG (0.5 nmol) (c) were injected. In vivo bioluminescence of Luci-A549 tumors after intraperitoneally injection of $2 \mathrm{mg} \mathrm{D}$-Luciferin substrate solution (Right column); ROI analysis of tumor-to-normal tissue (d) and liver-to-normal tissue ratios (e) of Cy5.5-mAb109 with (red line) and without (blue line) excess unlabeled mAb109 as block, and Cy5.5-lgG (green) in Luci-A549 tumor-bearing mice at $2 \mathrm{~h}$ to $7 \mathrm{~d}$ p.i

demonstrated high uptake and precise localization for tumors of the Cy5.5-mAb tracer at $2 \mathrm{~d}$ after administration.

Immunohistological study of human lung adenocarcinoma tissues To further confirm that mAb109 can be used for detection of human lung adenocarcinoma, we performed histological studies of human lung tumor tissues using mAb109 as the primary antibody. Typical IHC staining is shown in Fig. $6 ; 0.0087 \mathrm{mg} / \mathrm{mL}$ $(58 \mathrm{nM}) \mathrm{mAb} 109$ was selected as the primary antibody, and general polyperoxidase-anti-mouse/rabbit lgG was selected as the second antibody. Strong stain signals (deep yellow) were clearly observed around the nuclide (blue).

Accordingly, patients with lung adenocarcinoma were selected who had available tissue sections and demographical data (Table 1). The characteristics of a cohort of 12 lung adenocarcinoma tissues are presented in Table 1. Representative immunohistochemistry staining revealed that heavy expression of the mAb109-related receptor was higher in human lung adenocarcinoma tissues. Eleven of 12 tissues expressed some level of mAb109-related receptor. By contrast, 7/12 tissues overexpressed the receptor, reaching a score of 3 .

\section{DISCUSSION}

There is a statistically significant correlation among the expression of Prdx 1 and tumor cell proliferation, recurrence, and progression in lung patients ${ }^{[9-13]}$. We previously reported that the ${ }^{111}$ In-labeled mAb109 antibody may be useful for Prx I overexpression tumor imaging ${ }^{[15,16]}$. Therefore, in this study, a novel antibody, mAb109, was validated, modified, and characterized in a subcutaneous human lung adenocarcinoma A549 xenograft model and human lung tissues to determine its potential for lung cancer diagnosis. This study was performed to establish and validate Prdx1 for noninvasive PET imaging of lung adenocarcinoma. The production of the mAb109 antibody used activated B cells harvested from spleen tissue of ZR 75 cell-immunized BALB/c mice. Over a $99 \%$ protein purity and $150 \mathrm{kDa}$ molecular weight of the novel antibody were confirmed by SDS-PAGE and MALDI-TOF analysis, respectively. Western blot analysis showed that the A549 cell line had high Prdx expression (Fig. 1d, e).

To confirm the binding sensitivity of this novel antibody to lung adenocarcinoma tissues, mAb109 was diluted to 580, 145, 97, and $58 \mathrm{nmol}$ (1:50-1:500 dilution) using a primary antibody for IHC staining. As shown in Fig. $7 a-c$, it was too heavy to determine the 
a

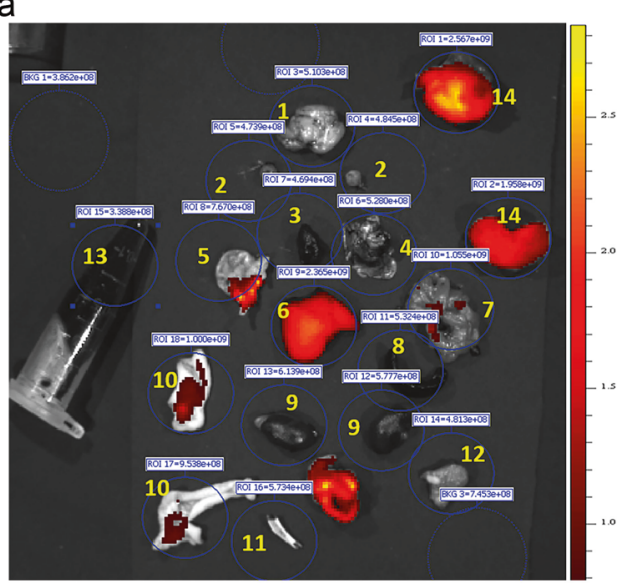

1. Brain

2. Eyes

3. Heart

4. Lung

5. Stomach

6. Liver

7. Intestine

8. Spleen

9. Kidney

10. Skin

11. Bone

12. Muscle

13. Blood

14. Tumor

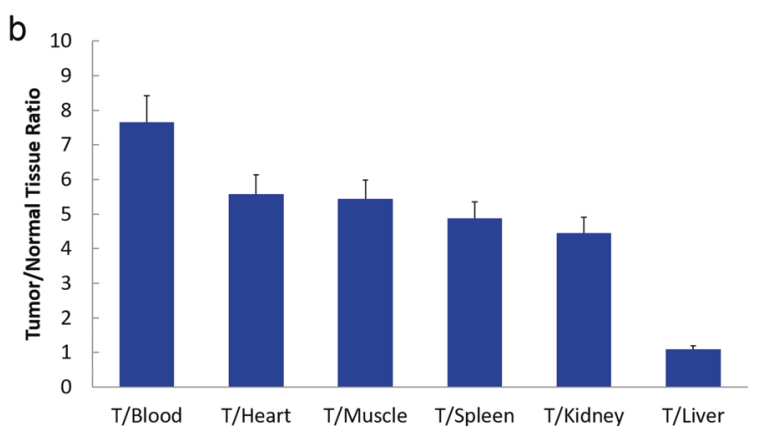

C

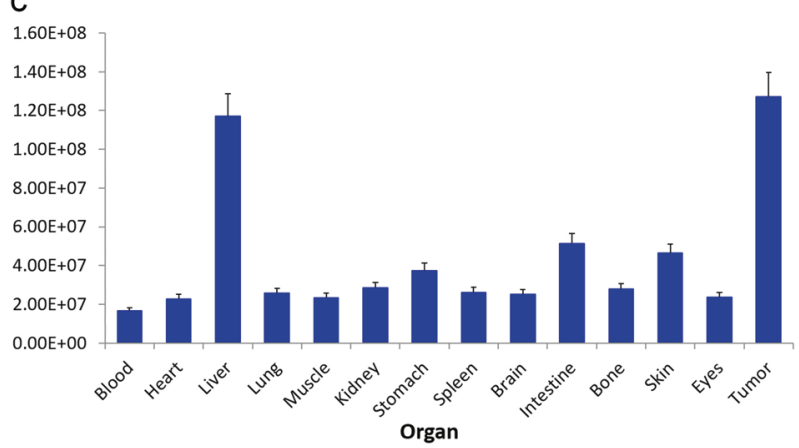

Fig. 5 a Ex vivo imaging of tumor and normal tissues of Cy5.5mAb109 at $48 \mathrm{~h}$ p.i. b Fluorescence intensity ratios of tumor-tonormal tissues based on the ROI analysis. (c) ROI analysis of fluorescent signal from tumor and normal tissues. Error bar was calculated as the standard deviation $(n=3)$

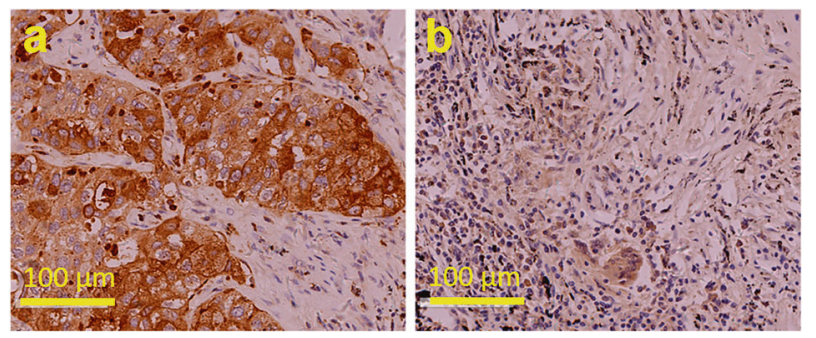

Fig. 6 Typical picture of Immunohistochemical (IHC) analysis of human lung adenocarcinoma tissue sample (a) and lymph node sample (b) using $58 \mathrm{nM}$ mAb109 as primary antibody

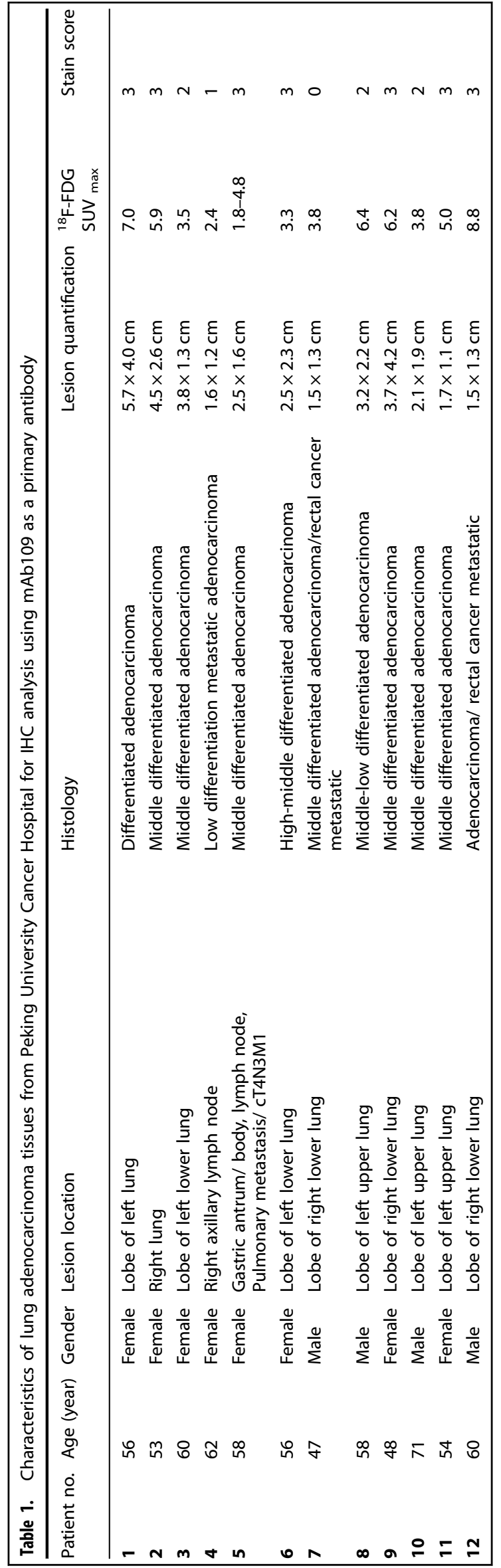



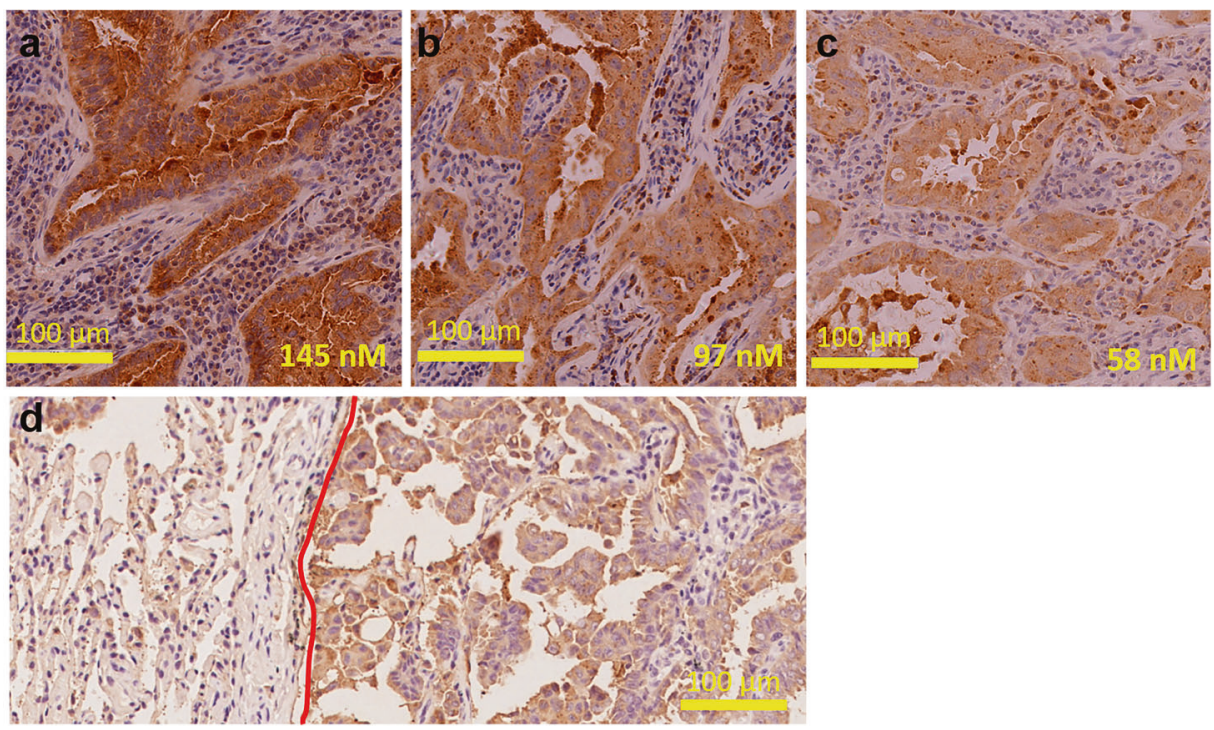

Fig. 7 Each concentration $145 \mathrm{nM}(\mathbf{a}), 97 \mathrm{nM}(\mathbf{b})$ and $58 \mathrm{nM}$ (c) of mAb109 used as a primary antibody for stain the same human lung tumor samples. d Typical picture of IHC analysis of human lung tumor samples using mAb109 as primary antibody. (Red curve distinguishes the normal tissue (left) and adenocarcinoma (right))

actual pathologic state of tumor tissue, while with more than $145 \mathrm{nmol}$ of mAb109, even $97 \mathrm{nM}$, IHC staining of lung tissue was difficult to recognize. With the decrease of mAb109 primary antibody concentration to $58 \mathrm{nM}$, the $\mathrm{IHC}$ staining results became reliable for predicting the actual state of the tumor.

MAb109 was further radiolabeled with the positron emitter radionuclide ${ }^{64} \mathrm{Cu}$ using a commercially available bifunctional chelator, NCS-Bz-NOTA, for noninvasive imaging of lung tumors. The binding affinity showed that the $\mathrm{Kd}$ value of ${ }^{64} \mathrm{Cu}$-NOTAmAb109 was approximately $29.6 \mathrm{nM}$ and that the Ki value was $10.6 \mathrm{nmol}$. The NIRF agent Cy5.5-mAb109 was synthesized for in vivo cell imaging. Cy5.5-mAb109 had a high level of fluorescence on the surface of A549 cells. To further evaluate the specific binding affinity of mAb109, the nonspecific contrast agent ${ }^{64} \mathrm{Cu}$-NOTA-lgG was introduced in cell uptake experiments. The cell uptake of ${ }^{64} \mathrm{Cu}$-NOTA-mAb109 reached $6.77 \% \pm 0.09 \%$ after $2 \mathrm{~h}$ of incubation compared with only approximately $2.0 \%$ for ${ }^{64} \mathrm{Cu}-\mathrm{NOTA}$-lgG. In addition, this binding was strongly blocked by an excess of unlabeled mAb109 antibody.

Then, an in vivo immune-PET/CT imaging study was performed on mice bearing A549 tumor xenografts (Figs. 3a and S2). ${ }^{64} \mathrm{Cu}-$ NOTA-mAb109 showed typical antibody distribution patterns in normal tissues, such as the liver and kidney. ${ }^{64} \mathrm{Cu}$-NOTA-mAb109 uptake in A549 tumors increased over time, and tumors were clearly visible at $24 \mathrm{~h}$ after injection until $60 \mathrm{~h}$. The blocked group did not show obvious tumor uptake. In addition, accumulation of ${ }^{64} \mathrm{Cu}$ NOTA-IgG in tumors was caused only by passive uptake, and rapid clearance was observed in the tumor model (Fig. S2). To further validate the PET results, mice were sacrificed at the last imaging time point to determine the ex vivo biodistribution. A549 tumor uptake of ${ }^{64} \mathrm{Cu}$-NOTA-mAb109, mAb109 block and ${ }^{64} \mathrm{Cu}-\mathrm{NOTA}$-lgG was 9.34 $\pm 0.67 \% \mathrm{ID} / \mathrm{g}, 2.29 \pm 0.47$ and $2.68 \pm 0.69 \% \mathrm{ID} / \mathrm{g}$, respectively. The significantly higher uptake of ${ }^{64} \mathrm{Cu}$-NOTA-mAb109 in tumor tissues compared to the other two groups indicates the specific lung cancer targeting of ${ }^{64} \mathrm{Cu}$-NOTA-mAb109. The autoradiography of each tumor tissue also confirmed this finding.

The tumor-targeting efficacy of modified mAb109 $(0.2 \mathrm{nmol}$ Cy5.5-mAb109) was also evaluated by static small-animal NIRF scans of mice bearing human lung adenocarcinoma cancer A549 tumors. Representative NIRF images are shown in Fig. 4. A549 xenografted tumors can be clearly visualized by the Cy5.5mAb109 tracer from the surrounding background tissue from
$24 \mathrm{~h}$ to as long as 16 days. Both the blocked group and the Cy5.5IgG injected group did not show such persistent and clear NIRF imaging, which indicates the specific binding of this mAb109 antibody. In addition, Fig. $3 \mathrm{~b} 2$ and Fig. $5 \mathrm{~b}$ show similar tumor/lung tissue ratios of up to 4.5 from the ex vivo gamma counter-based and fluoresce intensity-based biodistribution studies, indicating that modified mAb109 probes hold great potential for lung cancer imaging due to the high signal to background ratios for both PET and NIRF.

Using IHC staining studies, we isolated a cohort of 12 lung adenocarcinoma tissues from patient-resected solid tumors. Again, mAb109 was used as the primary antibody for this study. As shown in Fig. 7d, markedly stronger mAb109 staining was observed in lung adenocarcinoma tissues compared with adjacent normal tissues. We also confirmed that there was no relationship between the ${ }^{18} \mathrm{~F}-\mathrm{FDG}$ $\mathrm{PET} / \mathrm{CT}$ SUV $_{\text {max }}$-based semiquantitative results and mAb109 IHC score. Together, these findings indicate that labeled mAb109 may be useful for detecting lung adenocarcinoma.

The target antigens of the mAb109 antibody are not clearly identified in this study, which is a limitation of this research. However, with the implementation of this modified production approach, we were able to generate stable mAb109 antibody at a high yield. The resulting ${ }^{64} \mathrm{Cu}$-NOTA-mAb109/Cy5.5-mAb109 showed favorable imaging properties/specificity for imaging non-small lung cancer A549 tumors and high sensitivity in tumor cells, mice-based tumor tissues and human lung adenocarcinoma tissues. These results demonstrate that the mAb109 antibody can serve as a new platform for the development of novel agents for lung adenocarcinoma noninvasive imaging.

Herein, we demonstrated for the first time, to our knowledge, that the expression of $\operatorname{Prdx} 1$ can be noninvasively evaluated with PET imaging for potential patient selection. In summary, we produced a novel antibody, mAb109, and evaluated the feasibility of using a mAb109-based PET probe for lung cancer imaging. Clinical translation studies of the application of mAb109-based imaging probes for monitoring lung adenocarcinoma still need to be performed.

\section{CONCLUSIONS}

Here, we report the production of a new antibody, mAb109, that targets human lung adenocarcinoma. Initially, mAb109 was 
radiolabeled with the positron emitter ${ }^{64} \mathrm{Cu}$, and in vitro cell uptake experiments confirmed its binding affinity to A549 cells. Noninvasive uptake of ${ }^{64}$ Cu-NOTA-mAb109/Cy5.5-mAb109 was evaluated by high-resolution micro-PET/C and NIRF images in mice bearing A549 tumor xenografts. An immunohistological study of human lung tissues indicated that mAb109 can be used as a primary antibody to distinguish adenocarcinoma. Over $90 \%$ of all lung cancer patients express some level of mAb109-related receptor. With further optimization and development, mAb109based PET tracers hold potential for use in noninvasive lung adenocarcinoma diagnosis.

\section{ACKNOWLEDGEMENTS}

This work was financially supported by National Natural Science Foundation of China projects (Nos. 81671733 and 81571705), Natural Science Foundation of Beijing Municipality (No. 7171002), the Beijing Nova program (No. Z171100001117020), and the Young Top-Notch Talents Team program of Beijing Excellent Talents Funding (No. 2017000021223ZK33).

\section{AUTHOR CONTRIBUTIONS}

$\mathrm{HZ}, \mathrm{ZC}$, and $\mathrm{ZY}$ designed the experiments. $\mathrm{HZ}$, TLL, CHL, JW, HZ, BD, JS, CKZ, and ZFL performed all the experiments and analyzed the results. $\mathrm{HZ}, \mathrm{TLL}, \mathrm{ZC}$, and $\mathrm{ZY}$ wrote the paper, and all the authors read the paper.

\section{ADDITIONAL INFORMATION}

The online version of this article (https://doi.org/10.1038/s41401-019-0294-9) contains supplementary material, which is available to authorized users.

Competing interests: The authors declare no competing interests.

\section{REFERENCES}

1. Chen WQ, Zheng RS, Baade PD, Zhang SW, Zeng HM, Bray F, et al. Cancer statistics in China, 2015. CA Cancer J Clin. 2016;66:115-32.

2. Zhou CC. Lung cancer molecular epidemiology in China: recent trends. Trans Lung Cancer Res. 2014;3:270-9.
3. Zou XN, Lin DM, Wan X, Chao A, Feng QF, Dai Z, et al. Histological subtypes of lung cancer in Chinese males from 2000 to 2012. Biomed Environ Sci. 2014;27:3-9.

4. Siegel RL, Miller KD, Jemal A. Cancer statistics, 2015. CA Cancer J Clin. 2015; 65:5-29.

5. Abouzied MM, Crawford ES, Nabi HA. ${ }^{18}$ F-FDG imaging: pitfalls and artifacts. J Nucl Med Technol. 2005;33:145-55.

6. Selzner M, Hany TF, Wildbrett P, McCormack L, Kadry Z, Clavien PA. Does the novel PET/CT imaging modality impact on the treatment of patients with metastatic colorectal cancer of the liver? Ann Surg. 2004;240:1027-36.

7. Weiner GJ. Building better monoclonal antibody-based therapeutics. Nat Rev Cancer. 2015;15:361-70.

8. Rhee SG, Kang S, Chang TS, Jeong W, Kim K. Peroxiredoxin, a novel family of peroxidases. IUBMB Life. 2001;52:35-41.

9. Lehtonen ST, Svensk AM, Soini Y, Pääkkö P, Hirvikoski P, Kang SW, et al. Peroxiredoxins, a novel protein family in lung cancer. Int J Cancer. 2004;111:514-21.

10. Kim JH, Paul NB, Baek SH, Ramnath N, Liang P, Kim HR, et al. Up-regulation of peroxiredoxin 1 in lung cancer and its implication as a prognostic and therapeutic target. Clin Cancer Res. 2008;14:2326-33.

11. Woo HA, Yim SH, Shin DH, Kang D, Yu DY, Rhee SG. Inactivation of peroxiredoxin by phosphorylation allows localized $\mathrm{H}_{2} \mathrm{O}_{2}$ accumulation for cell signaling. Cell. 2010;140:517-28.

12. Chang JW, Jeon HB, Lee JH, Yoo JS, Chun JS, Kim JH, et al. Augmented expression of peroxiredoxin I in lung cancer. Biochem Biophys Res Commun. 2001;289:507-12.

13. Kim JH, Bogner N, Ramnath P, YoorimPark N, Yu J, Park YM. Elevated peroxiredoxin 1, but not NF-E2-related factor 2, is an independent prognostic factor for disease recurrence and reduced survival in stage I non-small cell lung cancer. Clin Cancer Res. 2007;13:3875-82.

14. Jadvar H. FDG PET in prostate cancer. PET Clin. 2009;4:155-61.

15. Lin ZH, Zhang H, Lin XF, Li ZF, Yang Z. Synthesis and evaluation of In-111-DOTAmAb109 monoclonal antibody for potential SPECT molecular imaging. Acta Chim Sin. 2015;73:36-40.

16. Wadas TJ, Wong EH, Weisman GR, Anderson CJ. Coordinating radiometals of copper, gallium, indium, yttrium, and zirconium for PET and SPECT imaging of disease. Chem Rev. 2010;110:2858-902.

17. Wu YM, Zhu H, Zhang B, Liu F, Chen JX, Wang YF, et al. Synthesis of site-specific radiolabeled antibodies for radioimmunotherapy via genetic code expansion. Bioconjug Chem. 2016;27:2460-8.

18. Miranda E, Nordgren IK, Male AL, Lawrence CE, Hoakwie F, Cuda F, et al. A cyclic peptide inhibitor of HIF-1 heterodimerization that inhibits hypoxia signaling in cancer cells. J Am Chem Soc. 2013;135:10418-25. 